\title{
Coexistence Curve of a Star-Shaped Polymer Solution. Comparison with the Hybrid Theory
}

\author{
Naoko Numasawa and Mamoru OKadA ${ }^{* \dagger}$ \\ School of Dentistry, Nihon University, \\ Surugadai, Chiyoda-ku, Tokyo 101-8310, Japan \\ * Department of Polymer Chemistry, Tokyo Institute of Technology, \\ Ookayama, Meguro-ku, Tokyo 152-8552, Japan
}

(Received August 3, 1998)

\begin{abstract}
KEY WORDS Star-Shaped Polymer / Coexistence Curve / Hybrid Theory / Polymer Solution / Phase
Diagram /
\end{abstract}

In a dilute polymer solution, polymer segments are not uniformly distributed in space because they are connected with chemical bonds. Such non-uniform distribution of local polymer segment density is of essential importance to thermodynamic properties of a dilute solution, while in a concentrated solution connectivity of segments reduces its influence on thermodynamic properties and the mean-field approximation is applicable to theoretical treatment of the solution. Phase equilibrium of a binary polymer solution is a phenomenon extending over these two concentration regimes and consequently the mean-field type theory such as the Flory-Huggins theory fails in quantitative description of phase diagram of a binary polymer solution. On the other hand, theories of dilute polymer solutions such as the virial expansions deteriorate their validity with increasing polymer concentration and cannot afford to give a phase diagram. To evade this difficulty, Koningsveld et al. ${ }^{1}$ proposed a theory in which the Flory-Huggins theory and the dilute polymer solution theory are combined so that the Flory-Huggins free energy crosses over to the free energy given by the virial expansion as concentration is decreased. Their theory, referred as the hybrid theory, was applied to linear polymer solutions and gave a good agreement with experimental results.

Recent experimental studies on phase diagrams of star-shaped polymer solutions revealed that phase diagram of a star-shaped polymer solution differs from that of the homologous linear polymer of the same molecular weight. ${ }^{2-4}$ Such solubility difference caused by molecular architecture cannot be explained either by the Flory-Huggins theory. However, it is expected that the hybrid theory reproduces the observed difference of phase diagrams of these polymer solutions, if the difference in free energy of dilute phase is a principal origin of the difference in phase diagram. Since explicit expressions of the second virial coefficients of starshaped polymer solutions have been given by the perturbation theories, extension of the hybrid theory to star-shaped polymer solutions is straightforward.

In this note, we calculated phase diagram of a binary star-shaped polymer solution based on the hybrid theory

\footnotetext{
† To whom correspondence should be addressed
}

and compared with experimental results in order to examine validity of the hybrid theory.

\section{RESULTS AND DISCUSSION}

A binary polymer solution at temperature $T$ and polymer volume fraction $\phi_{2}$ is considered. The solution consists of a solvent and an $f$-arm star-shaped polymer of the total segment number $N$. According to the hybrid theory ${ }^{1}$ chemical potentials of solvent $\Delta \mu_{1}$ and polymer $\Delta \mu_{2}$ are given by

$$
\begin{gathered}
\Delta \mu_{1} / k T=\log \phi_{1}+(1-1 / N) \phi_{2}+\xi_{1} \phi_{2}^{2} \\
\Delta \mu_{2} / k N T=(1 / N) \log \phi_{2}+(1-1 / N) \phi_{1}+\xi_{2} \phi_{1}^{2}
\end{gathered}
$$

respectively, where $\phi_{1}=1-\phi_{2}, k$ is the Boltzmann constant and

$$
\begin{aligned}
\xi_{1}= & \alpha+\beta(1-\gamma)\left(1-\gamma \phi_{2}\right)^{-2}+\left(1 / 2-\chi_{1}\right)[1-h(z)] \\
& \times(1+\lambda)^{-1}\left(1+\lambda \phi_{1}\right) \exp \left(-\lambda \phi_{2}\right) \\
\xi_{2}= & \alpha+\beta\left(1-\gamma \phi_{2}\right)^{-2}+\left(1 / 2-\chi_{1}\right)[1-h(z)] \\
& \times(1+\lambda)^{-1}\left(1-\lambda \phi_{2}\right) \exp \left(-\lambda \phi_{2}\right)
\end{aligned}
$$

In eq 3 and $4, \alpha, \beta, \gamma$ are coefficients of temperature and concentration dependence of the interaction parameter $\chi$ in sufficiently concentrated solutions:

$$
\chi=\alpha+\beta\left(1-\gamma \phi_{2}\right)^{-1}, \quad \beta=\beta_{0}+\beta_{1} T^{-1}
$$

and $\chi_{1}$ is given with these coefficients by

$$
\chi_{1}=\alpha+(1-\gamma) \beta
$$

The coefficient $\lambda$ is a parameter proportional to $N^{1 / 2}$ and is set to the inverse of the overlap concentration $\phi_{2}^{*}$ in this study as first proposed by Einaga et al. ${ }^{5}$

$$
\lambda=1 / \phi_{2}^{*}
$$

The function $h(z)$ of the excluded volume parameter $z$ is related to the second virial coefficient $A_{2}$ as

$$
A_{2} V_{1} / v_{2}^{2}=\left[(1 / 2)-\chi_{1}\right] h(z)
$$

where $V_{1}$ the molar volume of the solvent and $v_{2}$ the specific volume of the polymer. For normal star-shaped polymer with $f$ arms $h(z)$ was calculated to the first order of $z$ by Casassa ${ }^{6}$ : 


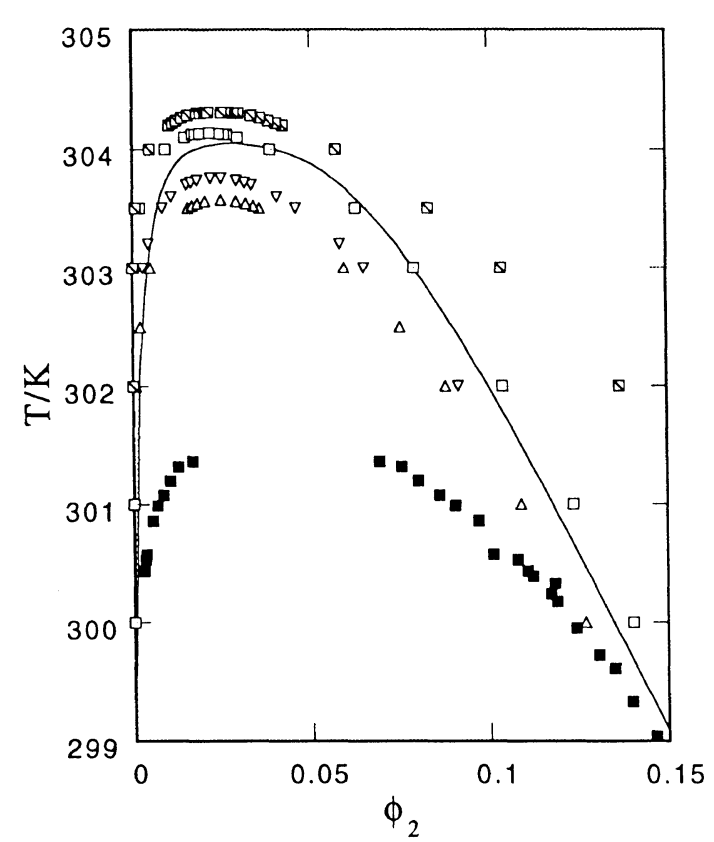

Figure 1. Theoretical and experimental coexistence curves of 11arm star-shaped polystyrene/cyclohexane solution and linear polystyrene/cyclohexane solution. Molecular weight of polymers is $2.05 \times 10^{6}$ : Theoretical with $\phi_{2}^{*}$ defined by eq $10 \mathrm{a}(\square)$ star, $(\triangle)$ linear; with $\phi_{2}^{*}$ defined by eq $10 \mathrm{~b}(\square)$ star, $(\nabla)$ linear; experimental $(\square)$ star, (-) linear.

$$
\begin{aligned}
& h(z)=1-C z+\cdots, \\
& C=\frac{32}{15 f^{1 / 2}}\left[7-4 \cdot 2^{1 / 2}+(f-1)\left(17 \cdot 2^{1 / 2}-9 \cdot 3^{1 / 2}-8\right)\right]
\end{aligned}
$$

Figure 1 shows experimental and theoretical coexistence curves of 11 -arm $(f=11)$ star-shaped polystyrene/ cyclohexane and linear $(f=1)$ polystyrene/cyclohexane solutions. Weight average molecular weight of starshaped polystyrene was $2.05 \times 10^{6}$ and experimental curve for linear polystyrene of the same molecular weight was obtained by the interpolation of the experimental data by Nakata et al. ${ }^{7}$ The theoretical curves were calculated from eq 1-9: the parameters for polystyrene/cyclohexane solutions were evaluated to be $\mathrm{e}^{1,8}$

$$
\begin{aligned}
& \alpha=-0.1597, \quad \beta_{0}=0.4987, \quad \beta 1=111.74 \mathrm{~K}, \quad \gamma=0.2365 \\
& V_{1}=108 \mathrm{~cm}^{3} \mathrm{~mol}^{-1}, \quad v_{2}=0.925 \mathrm{~cm}^{3} \mathrm{~g}^{-1} \\
& z=0.305\left(0.5-\chi_{1}\right) N^{1 / 2}
\end{aligned}
$$

The segment size $l^{3}$ was chosen equal to the solvent volume, which leads to $N=1.76 \times 10^{4}$. The overlap concentration $\phi_{2}^{*}$ is conventionally defined by using the radius of gyration of a polymer chain $R_{\mathrm{g}}$ to be

$$
\phi_{2}^{*}=\frac{N l^{3}}{(4 \pi / 3) R_{\mathrm{g}}^{3}}
$$

Experimental values of $R_{\mathrm{g}}$ of star-shaped $^{3}$ and linear ${ }^{9}$ polystyrenes at the theta temperature were obtained to be $R_{\mathrm{g}}=23.9 \mathrm{~nm}$ and $41.8 \mathrm{~nm}$, respectively, and their temperature dependence was neglected.

In case of linear polystyrene (PS), good agreement between theoretical and experimental curves was obtained. However, in case of star-shaped PS, agreement was not good. The difference between experimental and theoretical upper critical solution temperatures (UCST) $T_{\mathrm{c}}$ was roughly $2.5 \mathrm{~K}$ in star-shaped PS in contrast to $0.5 \mathrm{~K}$ in linear PS. A more serious problem is a discrepancy observed in the direction of a shift of phase diagram by branching of the component polymer. The theory predicted that the coexistence curve of starshaped PS was located at higher temperatures than the curve of linear PS, being opposite to the experimental results.

It is known that there are several ways of defining the overlap concentration other than eq $10 \mathrm{a}$. We calculated coexistence curves based on a different definition

$$
\phi_{2}^{*}=\frac{N l^{3}}{R_{\mathrm{g}}^{3}}
$$

in order to know the influence of definition of $\phi_{2}^{*}$ or the dependence on the parameter $\lambda$. The results were shown in Figure 1. Though $\phi_{2}^{*}$ was increased about four times by the change of definition, the coexistence curve of linear PS scarcely changed over a wide concentration range, which confirmed the weak $\lambda$-dependence already reported by Koningsveld et al. ${ }^{1}$ Coexistence curve of starshaped PS was more sensitive to $\lambda$ especially on the concentrated-phase side. However, the change of $\lambda$ resulted in enlarging the two-phase region and deteriorated the agreement with the experimental result.

In the hybrid theory, $\chi$ parameters applicable to limited concentration ranges, namely dilute or concentrated solution, are linearly combined to express the interaction parameter for arbitrary concentration:

$$
\begin{aligned}
& \chi=P \chi_{\mathrm{dil}}+(1-P) \chi_{\mathrm{conc}} \\
& P=\exp \left(-\lambda \phi_{2}\right)
\end{aligned}
$$

It seems more straightforward to combine chemical potentials and we attempted to calculate the critical temperature $T_{\mathrm{c}}$ based on this approach.

$$
\Delta \mu_{1}=P \Delta \mu_{1, \mathrm{dil}}+(1-P) \Delta \mu_{1, \mathrm{conc}}
$$

where

$$
\begin{aligned}
& \Delta \mu_{1, \mathrm{dil}} / k T=-\phi_{2} / N-\left(1 / 2-\chi_{1}\right) h(z) \phi_{2}^{2} \\
& \Delta \mu_{1, \mathrm{conc}} / k T=\log \phi_{1}+(1-1 / N) \phi_{2}+\chi_{1} \phi_{2}^{2}
\end{aligned}
$$

The critical temperatures of star-shaped PS $(f=11)$ solution and of linear PS solution were evaluated to be $303.6 \mathrm{~K}$ and $300.9 \mathrm{~K}$, respectively, and modification of the theory brought about no improvement.

As stated in the introductory section, phase equilibrium of a binary polymer solution is a phenomenon extending over dilute and semidilute (or concentrated) regimes. Hence, the difference of coexistence curves of a star-shaped polymer solution and a linear polymer solution is caused by a difference of chemical potential of the concentrated phase as well. Because of the higher segment density around the center of a star-shaped polymer, average number of intermolecular contacts in a semidilute solution is reduced near its center compared with the outer region, which leads to the difference in the mixing enthalpy from linear polymer solutions. We showed that such enthalpy effect actually resulted in an appreciable change of coexistence curve by carrying out a simple theoretical calculation. ${ }^{10}$ The theoretical 


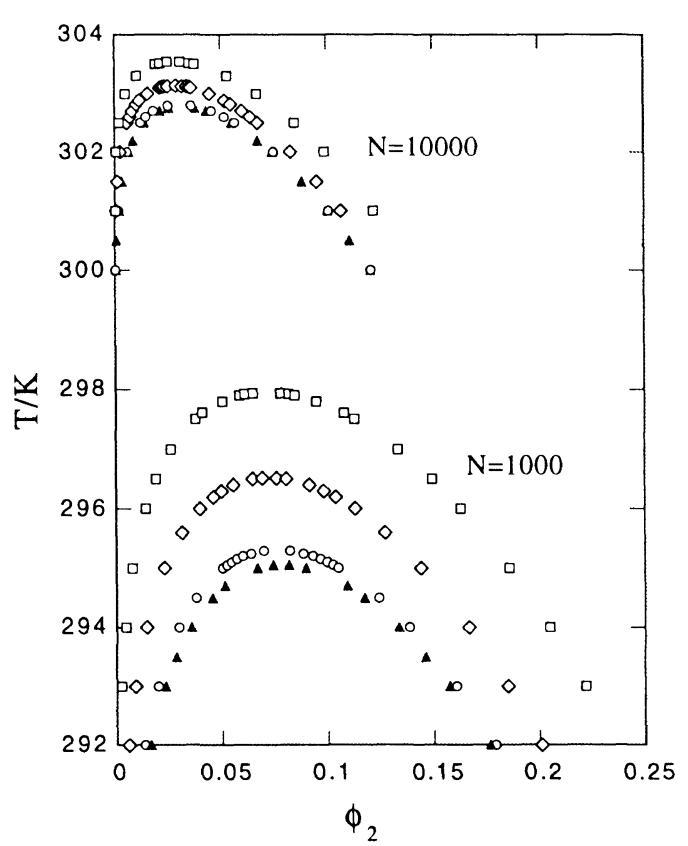

Figure 2. Coexistence curves, calculated by the hybrid theory, of cyclohexane solutions of star-shaped polystyrene with arm numbers $f=3(\bigcirc), 6(\diamond)$, and $11(\square)$ and linear $(\mathbf{A})$ polystyrene for segment numbers $N=1000$ and $N=10000$.

calculation correctly gave a result that the upper critical solution temperature (UCST) of 11-arm star-shaped $\mathrm{PS} /$ cyclohexane solution was lower than the UCST of linear PS/cyclohexane solution of the same molecular weight though it quantitatively overestimated the difference $\Delta T_{\mathrm{c}}$ of these two critical temperatures (theoretical $\Delta T_{\mathrm{c}}=8 \mathrm{~K}$, experimental $\left.\Delta T_{\mathrm{c}}=3 \mathrm{~K}\right){ }^{10}$

However, this enthalpy effect in the semidilute regime is considered to appear only in the case of relatively large number of arms $f$, and contribution of the effect is expected to be negligibly small for a 3-arm star-shaped polymer solution. Experimental results showed that even for $f=3$ the UCST is slightly lower than that of linear polymer solution. ${ }^{2}$ This observed difference must consequently arise from the free-energy difference in the dilute phase. As shown in Figure 2, application of the hybrid theory to 3-arm star-shaped PS solutions gave results opposite to the experimental observations, and the present calculation (eq 1-10) could not correctly describe the contribution of free energy of the dilute phase. Since the basic idea of the hybrid theory, i.e., connecting free energies of dilute and concentrated solutions with using a function that abruptly changes from unity to zero around the overlap concentration, seems reasonable, it is considered that the expression used for chemical potential of the dilute phase is not appropriate. Probably the higher virial terms should be taken into account.

\section{REFERENCES}

1. R. Koningsveld, W. H. Stockmayer, J. W. Kennedy, and L. A. Kleintjens, Macromolecules, 7, 73 (1974).

2. J. M. G. Cowie, A. Horta, I. J. McEwen, and K. Prochazka, Polym. Bull., 1, 329 (1979).

3. H. Yokoyama, A. Takano, M. Okada, and T. Nose, Polymer, 32, 3218 (1995).

4. M. Okada, A. Takano, and J. Iwai, 36th IUPAC Intnl. Symp. Macro., 1996, p 276.

5. Y. Einaga, S. Ohashi, Z. Tong, and H. Fujita, Macromolecules, 17, 527 (1984)

6. E. F. Cassasa, J. Chem. Phys., 37, 2176 (1962)

7. M. Nakata, N. Kuwahara, and M. Kaneko, J. Chem. Phys., 62, 4278 (1975).

8. J. Brandrup and E. H. Immergut, Ed., "Polymer Handbook," 3rd ed, Wiley Interscience, New York, N.Y., 1989.

9. B. K. Varma, Y. Fujita, M. Takahashi, and T. Nose, J. Polym. Sci., Polym. Phys. Ed., 22, 1781 (1984).

10. M. Okada and N. Numasawa, Comput. Theoer. Polym. Sci., 8, 259 (1998). 